\title{
3.2 Selbstorganisation von Organoiden aus Entodermzellen ${ }^{6}$
}

Zusammengefasst und aus dem Englischen übersetzt von Anja Pichl

Organoide sind dreidimensionale (3-D) biologische Systeme, die dafür verwendet werden, die Entwicklung, Homöostase (Aufrechterhaltung des Gleichgewichtszustandes durch innere Regulierungsvorgänge), Regeneration und Erkrankung von Organen in vitro zu modellieren; sie versprechen zudem therapeutischen Nutzen. Organoide spiegeln die Entwicklung in vivo wider und werden aus Gewebezellen (adulten Stammzellen) oder pluripotenten Stammzellen hergestellt. Die zu Beginn noch einheitlichen Ausgangszellen werden durch Signale aus dem Nährmedium und von individuellen Zellen zur Selbstorganisation angeregt, wobei die zunehmend differenzierten Zellen Strukturen bilden, die hinsichtlich ihrer Morphologie und oft auch Funktionalität dem Herkunftsgewebe ähneln. Organoide komplementieren herkömmliche zweidimensionale In-vitro-Kulturen und In-vivo-Tiermodelle der Entwicklung, insofern sie die experimentelle Kontrolle und Flexibilität von In-vitro-Methoden mit dem dreidimensionalen Kontext von In-vivo-Modellen verbinden, wobei zudem weniger ethische Einschränkungen bestehen als bei der Arbeit an Mensch oder Tier. Jedoch stehen wir bei der Verwendung von Organoiden noch ganz am Anfang des Verständnisses davon, wie externe Bedingungen und Signale zwischen einzelnen Zellen auf zellulärer Ebene das Auftreten von komplexen Strukturen fördern. In dieser Zusammenfassung stehen Organoide, die aus entodermalem ${ }^{7}$ Gewebe gewonnen wurden, im Fokus, wie z. B. der Lunge und Luftröhre, Leber, Bauchspeicheldrüse, Speiseröhre, Prostata, Speicheldrüse, Blase, Schilddrüse, des Gallengangs, Darms, und Magens (siehe Abbildung 1). Diskutiert

6 Dieser Beitrag ist eine deutsche Zusammenfassung des Review-Artikels „Self-organization of organoids from endoderm-derived cell“" von Allison Lewis, Rashmiparvathi Keshara, Yung Hae Kim und Anne Grapin-Botton, der 2020 im Journal of Molecular Medicine erschienen ist und eine umfangreiche Darstellung des wissenschaftlichen Sachstands sowie der aktuellen Literatur zum Thema (Stand Sommer 2020) bietet.

7 Während der Embryonalentwicklung entstehen im Embryo die drei Keimschichten, auch Keimblätter genannt: Ektoderm (Außenschicht), Mesoderm (Mittelschicht) und Entoderm (Innenschicht). Jedes dieser Keimblätter bildet bestimmte Gewebe und Organe aus. 
Abbildung 1: Entodermbasierte Organoide

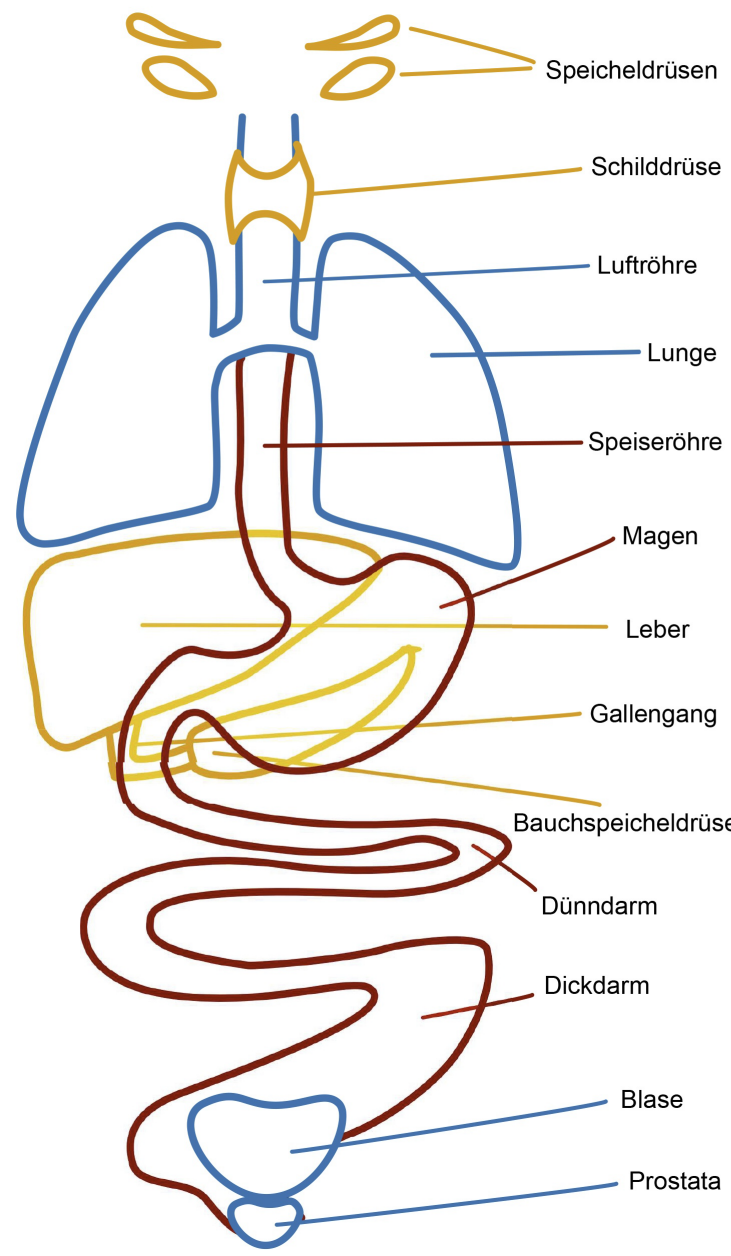

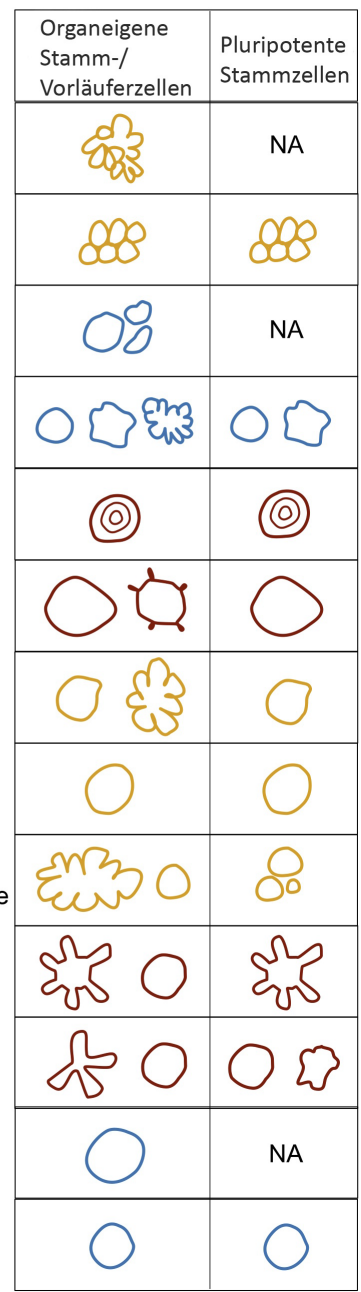

werden insbesondere die Ausgangsbedingungen der Zellen, Signalmechanismen und externe Kulturmedien, die die Entstehung von komplexer Selbstorganisation ermöglichen. Bahnbrechende Studien zu Organoidsystemen sowohl aus embryonalen als auch adulten Stammzellen haben den Weg für viele andere Studien geebnet, welche die Selbstorganisation von komplexen Strukturen durch die Bestandteile des Kultur- 
mediums erforschen und dabei das Wissen über Signalwege in der Entwicklung und Homöostase einbeziehen.

\subsubsection{Selbstorganisation}

Selbstorganisation ist im Allgemeinen ein Prozess, bei dem lokale Interaktionen zwischen Teilen eines zunächst ungeordneten Systems zur Bildung von komplexeren Strukturen (Strukturen höherer Ordnung) führen. Dieses Konzept, das in der Physik, der Verhaltensforschung bei Tieren, der Biologie und den Sozialwissenschaften Anwendung findet, wird häufig auch Organoiden zugeschrieben und kann diesbezüglich folgendermaßen reformuliert werden: Selbstorganisation ist ein Prozess, bei dem lokale Interaktionen zwischen Zellen, die zunächst ungeordnet sind, zur Entstehung von Mustern und Funktionen in komplexeren Strukturen, den Organoiden, führen (siehe auch Fagan, Kap. 4, für eine philosophische Diskussion des Konzepts der Selbstorganisation im Kontext der Organoidforschung). Diese Organisation wird weder von einer einzelnen Zelle noch durch externe Kontrolle vorangetrieben. Es wird angenommen, dass die Selbstorganisation Einfluss auf alle Komponenten bzw. Zellen des Systems ausübt. Dieser Einfluss macht das System robust gegenüber Störungen und ermöglicht die Aufrechterhaltung der Homöostase und Regeneration. Die Selbstorganisation kann jedoch durch die Wahl bestimmter Rahmenbedingungen beeinflusst werden, was meist durch eine Kontrolle der Bestandteile der Kulturmedien durch die Forschenden geschieht. Aber auch die intrinsischen Eigenschaften der Ausgangszellen steuern den Prozess. Eine weitere Kontrollebene kann durch die Beeinflussung der räumlichen Voraussetzungen und die Verwendung verschiedener Zellumgebungen wie Matrigel oder ähnlicher Alternativen erreicht werden. So kann z. B. die Entwicklung zweier Arten von Organoiden wie Vorder- und Mitteldarmorganoide und ihr Nebeneinanderplatzieren zur Bildung einer neuen, selbstorganisierten Struktur an der Schnittstelle führen, wodurch die Leber, Bauchspeicheldrüse und Galle betreffende Region gebildet wird. Im Folgenden werden aus dem Entoderm gewonnene Organoidsysteme vorgestellt und Fragen diskutiert, die für das gesamte Feld der Organoidforschung relevant sind: Welche Zelltypen haben die Fähigkeit zur Selbstorganisation? Welcher Austausch von Signalen zwischen Zellen und welches Ausmaß an Interaktionen sind notwendig, um die Selbstorganisation zu initiieren und den Prozess weiterzuentwickeln? Welche Art von externen Kontrollen erleichtern die Selbstorganisation von Organoiden? 


\subsubsection{Die zunächst ungeordneten Zellen}

Wichtig für das Verständnis der Selbstorganisation ist eine gute Beschreibung der ursprünglichen Bestandteile des Systems, der Zellen. Die Ausgangszellen, aus denen entodermale Organoide entstehen können, sind sehr verschieden. Dabei kann es sich um Stammzellen oder Vorläuferzellen aus adulten oder fetalen Organen handeln oder um pluripotente Stammzellen, die in vitro die Identität spezifischer Zellen aus entodermalen Organen erlangen können. In einigen Fällen sind Primärzellen mit der - inhärenten oder erst in vitro aufgetretenen - Fähigkeit zur Proliferation (Wucherung des Gewebes durch Zellvermehrung) zur Herstellung von Organoiden verwendet worden. Faszinierend ist, dass aus ihrem Zellverband gelöste Einzelzellen, entweder für sich oder als neu zusammengesetzte Gruppe, miteinander interagieren und so Gewebe- oder organähnliche Strukturen bilden können. Dies übersteigt bei Weitem die Fähigkeit einer herkömmlichen Gewebeprobe (Explantat) unter Kulturbedingungen erhalten zu werden.

\section{Organoide aus adulten Stammzellen, Vorläuferzellen oder jeglichem Zelltyp mit} Proliferationspotenzial

Das am ausführlichsten untersuchte Organoidsystem auf Basis adulter Stammzellen und Vorläuferzellen sind Darmorganoide, die entweder aus bestimmten adulten Stammzellen des Darms oder aus ganzen Darmkrypten (in Einstülpungen der Dünndarmschleimhaut sitzendes Drüsengewebe, welches Stammzellen besitzt) in vitro gebildet werden. Viele Organoide können aus einer einzigen Zelle gebildet werden, wenn von primären Zellen ausgegangen wird, aber die Wahrscheinlichkeit der Bildung von Organoiden steigt, wenn zwei oder mehr Zellen zusammen kultiviert werden. In der Regel sind einzelne Zellen, wenn sie gerade aus dem Gewebe isoliert wurden, zunächst bei der Bildung von Organoiden ineffizient und werden erst später potent. Darüber hinaus ist für die Bildung von Organoiden bei einigen Organen das Vorhandensein anderer Zelltypen erforderlich. Wenn sich das System nur aus einer Zelle entwickelt, entstehen weitere Komponenten im System, indem sich diese und ihre Tochterzellen teilen. Dies unterscheidet Organoide von vielen anderen selbstorganisierten Systemen wie z. B. Vogelschwärmen, bei denen die Anzahl der Komponenten im System stabil bleibt. Darüber hinaus werden die Zellen in einigen Organoidsystemen so vermehrt, dass die Tochterzellen in einem ähnlichen Zustand verbleiben, während in anderen Systemen Asymmetrien mit Tochterzellen auftreten, die ganz unterschiedliche Eigenschaften haben. Die Bedingungen für die Entstehung von Asymmetrien sind ein spannendes Forschungsgebiet. 


\section{Organoide aus pluripotenten Stammzellen}

Entodermale Organoide aus pluripotenten Stammzellen werden in der Regel nach einer von Jason Spence (siehe Frum/Spence, Kap. 3.1) entwickelten Methode hergestellt, die sich von Methoden zur Herstellung von Organoiden aus anderen Keimschichten wie z. B. Hirn- oder Nierenorganoiden, unterscheidet. Statt ganze Aggregate von humanen embryonalen Stammzellen (hES-Zellen) als Ausgangsbasis zu nehmen und diese zur Differenzierung zu bewegen, werden die humanen pluripotenten Stammzellen (hPS-Zellen) zunächst einzeln in vitro kultiviert und ihre Differenzierung durch Signalmoleküle gesteuert. Nach einiger Zeit bilden sich 3-D-Kugeln aus Hinterdarmzellen in einem bislang noch nicht verstandenen Selbstorganisationsprozess. Werden diese Kugeln in Matrigel versetzt, entwickeln sie sich zu Darmorganoiden, die sowohl das Darmepithel (die Darmschleimhaut) als auch seine mesenchymale (aus Bindegewebe bestehende) Umgebung umfassen, mit villusartigen Bereichen (Ausstülpungen, wie sie typischerweise auf Zellen der Darmschleimhaut zu finden sind), die sich aus zum Darm gehörenden Stammzellen und allen dazugehörigen differenzierten Zelltypen zusammensetzen. Im Gegensatz zu aus Darmkrypten stammenden Organoiden enthalten diese hPS-Zell-abgeleiteten Organoidkulturen mit den erwähnten Bindegewebszellen auch Zellen mesodermalen Ursprungs. Organoide mit Bindegewebszellen sind potenziell Modellsysteme zum Verständnis von Interaktionen zwischen Epithel und Bindegewebe während der Organogenese, von Zellspezifizierungen sowie der Bestimmung der Gewebenische; dennoch scheint das Bindegewebe zur Bildung von Organoiden nicht erforderlich zu sein. Für andere Organoide können hPS-Zellen in 2-D in gewebespezifische (z. B. pankreatische) Vorläuferzellen differenziert und dann isolierte Zellen oder kleine Zellgruppen in Matrigel eingebettet und expandiert werden. Es gibt noch weitere Methoden zur Herstellung entodermstämmiger Organoide, beispielsweise die Herstellung entodermaler oder gewebespezifischer Zellen in 2-D und deren Kokultivierung mit gewebeeigenen mesenchymalen oder epithelialen Zellen. Für einige Organoide werden hPS-Zellen als 3-D-Aggregate kultiviert, sodass sie embryoide Körper („embryoid bodies“) bilden. Für Organoide der Speiseröhre konnte die Entstehung eines ausgeprägten, differenzierten Epithels aus ungeordneten embryoiden Körpern gezeigt werden. Diese Aufzählung zeigt, dass aus hPS-Zellen gewonnenes Entoderm in viele Gewebe direkt differenziert werden kann, auch wenn die methodischen Prinzipien je nach Lehrmeinung variieren. 


\subsubsection{Welche Art von Ordnung entsteht?}

\section{Entstehung räumlicher Ordnung}

Systeme werden als „emergent“ bezeichnet, wenn ihre Bestandteile bestimmte Eigenschaften haben, die die einzelnen Teile an sich nicht aufweisen. In diesem Sinn weisen Organoide emergente räumliche Organisationen auf wie z. B. auch Fisch- oder Vogelschwärme. Die meisten entodermalen Organoide bilden ein kugelförmiges Lumen (inneren Hohlraum), wobei die Struktur des auskleidenden Epithels unterschiedlich sein kann. Wichtig ist, dass diese unterschiedlichen Arten von Lumina In-vitro-Phänomene sind, die der Verschiedenheit der Lumina (und sie auskleidender Epithelzellen) in den verschiedenen Organen entsprechen. Warum Organoide solche verschiedenen Lumina bilden, muss noch erforscht werden. Die Lumenbildung ist ein Selbstorganisationsverhalten, da Zellen dies kollektiv, aber nicht einzeln tun.

Verästelungen oder Falten sind eine weitere Art der Struktur, die sich in Organoiden u. a. von Darm, Pankreas und Lunge beobachten lässt. So bilden beispielsweise differenzierte Darmorganoide faltenförmige Krypten zusammen mit Drüsenzellen (Panethzellen). Letztere zeigen sich noch vor den morphologischen Veränderungen, weshalb die Falten als Folge der Differenzierung betrachtet werden. Die Faltenbildung bei Organoiden bedarf jedoch noch genauerer Untersuchung. Experimente mit Hirnorganoiden legen nahe, dass das Zusammenspiel zwischen materiellen Eigenschaften des Organoids und der Umgebung, in der es heranwächst, ein entscheidender Faktor bei der Faltenbildung ist. Ein wichtiger Unterschied zwischen Organoiden und Organen in vivo liegt in der begrenzten Komplexität von Formen und deren gewöhnlich von allen Seiten gleichbleibender (isotroper) Natur. So bilden die meisten Darmorganoide bis auf wenige Ausnahmen Kugeln statt Röhren, die sich normalerweise im Darm finden. Durch das Zusammenfügen von Organoiden lassen sich, wie eingangs erwähnt, auch komplexere, nicht isotrope Formen entwickeln.

\section{Entstehung der Differenzierung}

Zusätzlich zur Morphogenese (Gestaltbildung) entstehen häufig neue Zelltypen in Organoiden. Die Differenzierung von Organoiden hängt weitgehend vom Potenzial der initial verwendeten Zellen sowie von der Zusammensetzung des Mediums ab. Diese Kulturmedien zu bestimmen ist ein wesentlicher Fokus der Organoidforschung. Sehr häufig werden die Medien so zusammengesetzt, dass sie entweder den noch undifferenzierten, teilungsfähigen Zustand einer Vorläuferzelle erhalten oder die Differenzierung vorantreiben. Diese unterschiedlichen Medien können zudem nacheinander eingesetzt werden, sodass zunächst das Wachstum des Organoids gefördert 
und dann seine Differenzierung kontrolliert wird. Wenn gewebespezifische Stammbzw. Vorläuferzellen verwendet werden, entsprechen die differenzierten Zelltypen weitgehend denen des ursprünglichen Organs, auch wenn die Relationen nicht immer übereinstimmen und manche Zelltypen (z. B. endokrine Zellen in Darm, Lunge und Prostata) schwer zu erhalten sind. Auch die Kombination verschiedener Zelltypen in einem einzelnen Organoid bleibt hinsichtlich einiger Organe, wie z. B. der Leber, eine Herausforderung. Einen Überblick über die Differenzierung in verschiedenen entodermalen Organoidsystemen bietet Abbildung 1.

Bei Organoiden auf Basis pluripotenter Zellen stellt sich das Problem des Auftretens „unerwünschter“ Zelltypen, das bisher noch unzureichend untersucht ist und durch die Verwendung der Einzelzellanalyse ${ }^{8}$ besser erforscht werden kann.

\section{Entstehung funktionaler Eigenschaften}

Organoide werden häufig in der Hoffnung hergestellt, dass sie als Modelle von Organfunktionen und ihrer krankheitsbedingten Beeinträchtigungen fungieren; daher ist das Auftreten emergenter funktionaler Eigenschaften ein wichtiges Merkmal. Bisher sind nur wenige Funktionen untersucht worden, z. B. die Pumpfunktion in Organioden des Darms, der Lunge, des Pankreas und Gallengangs und ihre Beeinträchtigung bei zystischer Fibrose. Viele weitere Funktionen müssen noch erforscht werden. Wichtig ist zudem der Vergleich mit der Organfunktion in vivo, um die Limitationen der Modelle einschätzen zu können. Insbesondere bei Organoiden auf Basis von hPS-Zellen wird der Differenzierungsprozess, der im Körper neun Monate dauert (Embryonalentwicklung in der Schwangerschaft), in vitro in wenigen Wochen rekapituliert, auch wenn eine Kultivierung über Monate inzwischen möglich wird. Ob der Prozess in vitro beschleunigt abläuft oder die Zellen ihrem natürlichen Rhythmus folgen und frühe fetale Zellen bleiben, ist eine Frage, die gerade erst von der Forschung untersucht wird.

\section{Entstehung von Organbereichen}

Bei Organoiden, die eine komplexe Organisation erlangen, lässt sich beobachten, dass Organbereiche in einer bestimmten räumlichen Anordnung in Mustern entstehen, die an die in vivo beobachteten erinnern; z. B. bilden sich Krypten, die Stammzellen

8 Siehe hierzu Walter/Schickl (2019): Einzelzellanalyse in Forschung und Medizin. Eine Stellungnahme der interdisziplinären Arbeitsgruppe Gentechnologiebericht. Kostenlos abrufbar unter: https:/ /www.gentechnologiebericht.de/fileadmin/user_upload/Webseitendateien/Dokumente/BBAW_Einz elzellanalyse_A5_PDF-A1-b.pdf [14.07.2020]. 
beherbergen, in Darmorganoiden, und Gruben mit Grubenzellen entwickeln sich in Magenorganoiden. Diese Prozesse können lokale Signalaktivitäten kombinieren, aber auch aus der Neuordnung von Zellen und Wechselwirkungen zwischen Zellen und dem sie umgebenden Gewebe resultieren. Ein verstärkter Einsatz von Live-Bildgebungsverfahren verspricht hierüber Aufklärung.

\subsubsection{Austausch von Signalen zwischen Zellen und Ausmaß der Interaktionen}

Ein wichtiges Prinzip der Selbstorganisation ist der Austausch von Informationen zwischen den Bestandteilen eines Systems. Kürzlich durchgeführte Experimente mit Darmorganoiden haben Licht auf die Signale geworfen, die Zellen nach Auflösung des Gewebes in Einzelzellen zur Selbstorganisation untereinander austauschen. Es wurde gezeigt, dass Drüsenzellen essenzielle Nischensignale für die Selbstorganisation von Krypten und Zotten bereitstellen, sowohl in vivo als auch in vitro. Wie oben erwähnt, können in vielen Systemen auch einzelne Zellen Organoide bilden, doch dies spricht nicht gegen die Wichtigkeit von Kooperation und Kommunikation zwischen Zellen. Bestandteile des Kulturmediums können den für die Selbstorganisation erforderlichen Bedarf an Signalaustausch ersetzen, indem sie Signale nachahmen, die normalerweise durch andere Zellen bereitgestellt werden. Für manche Organe kann auch die Zugabe von Nicht-Epithelzellen das Wachstum von Organoiden aus einzelnen Epithelzellen ermöglichen.

\subsubsection{Positive und negative Rückkopplungsschleifen}

Es ist noch ein weiter Weg von den wenigen Signalen, von denen wir wissen, dass sie zwischen Zellen ausgetauscht werden, zu einem Verständnis von Selbstorganisation und Emergenz (Auftreten neuer Eigenschaften durch Zusammenwirkung) in Organoiden. Von anderen Systemen, an denen Emergenz untersucht worden ist, lässt sich viel lernen, beispielsweise dass das bloße Vorhandensein von Interaktionen zwischen Zellen nicht ausreicht, um emergentes Verhalten zu garantieren; viele der Interaktionen könnten vernachlässigbar oder irrelevant sein, $z$. B. indem sie einander aufheben oder das Auftreten interessanten Verhaltens verhindern. Dementsprechend ist nicht die bloße Anzahl von Verbindungen zwischen Bestandteilen entscheidend, sondern wie diese Verbindungen organisiert sind. Eine hierarchische Organisation kann beispielsweise emergentes Verhalten hervorrufen. Ein verbreitetes Merkmal emergenter Systeme ist die Präsenz positiver und negativer Rückkopplungsschleifen. Bei einer positiven Rückkoppelung verstärkt ein Effekt sich selbst. Bei einer negativen Rückkoppelung 
hemmt der Effekt die ursprüngliche Reaktion. Im Allgemeinen stabilisieren negative Rückkopplungen Strukturen, wohingegen positive Rückkopplungen Veränderung begünstigen. In manchen Fällen muss das System eine bestimmte Stufe an Diversität, Organisation und Verbundenheit erreichen, bevor emergentes Verhalten in Erscheinung tritt. Von anderen Systemen ist auch bekannt, dass das Signal, das emergentes Verhalten auslöst, extern oder auch „noise“9 sein kann. Entsprechende theoretische Überlegungen sind wichtig für das Verständnis externer Kontrolle, beispielsweise in Form der Komponenten des Mediums und der Dauer der Exposition mit diesen Komponenten, um die Reaktion des Organoids zu steuern und reproduzierbarer zu machen.

\subsubsection{Externe Kontrolle}

Zellen in Organoiden reagieren nicht nur auf Signale, sondern erzeugen und verbreiten auch Signale, sowohl autonome als auch nicht-autonome. Während des Selbstorganisationsprozesses passen sich Zellen selektiv an die verfügbaren Signale an, die im Kulturmedium bereitgestellt werden. Die Mehrzahl der Kulturmedien für entodermale Organoide sind Medien, die Basisnährstoffe für den Zellerhalt in der Kultur liefern. In den meisten organoiden Systemen werden verschiedene Medien verwendet, um nacheinander die Proliferation und anschließend die Differenzierung zu fördern.

Organoide Kulturmedien bieten eine Auswahl an Signalmolekülen, und die Zellen in Organoiden nutzen diese je nach Bedarf während des Selbstorganisationsprozesses. Es wäre faszinierend zu untersuchen, wie diese homogenen Medienbestandteile selektiv von mehreren Zellen genutzt werden, um heterogene Zelltypen sowie eine variable Morphologie zu erzeugen.

Neben der Kontrolle durch das Medium und die Matrix bieten die jüngsten Entwicklungen von Organ-on-a-Chip-Systemen Plattformen, die sich auf die Erzeugung und Nutzung von Organoiden auswirken. Diese Systeme machen die Herstellung von Organoiden mit geringerer Größenvariabilität und den Einbau von Anzeigen für Funktionstests möglich, und sind nützliche Hilfsmittel für Medikamentenscreenings und Krankheitsmodellierung. Darüber hinaus werden ganze Body-on-a-Chip-Systeme etabliert, bei denen mehrere unterschiedliche Organoide in einem einzigen System zur Untersuchung der Wechselwirkungen und der Physiologie des gesamten Körpers miteinander verbunden werden.

9 Als Hintergrundrauschen oder Störgeräusche („noise“) bezeichnet man in der Wissenschaft unspezifische Signale, die herausgefiltert werden müssen, um relevante Signale erkennen zu können. 


\subsubsection{Schlussfolgerung und Ausblick}

In den letzten 10 Jahren sind Organoidsysteme für die meisten entodermalen Organe und verfeinerte Methoden für besser reproduzierbare Systeme entwickelt worden. In den kommenden Jahren wird die Entwicklung einer den körpereigenen Organen ähnlicheren Architektur sowie die Eingliederung verschiedener Zelltypen - einschließlich der Gefäße - und von automatisierten Kultursystemen erwartet. Gleichzeitig ist ein umfassender Vergleich mit körpereigenen Organen notwendig, um die Aussagekraft und Bedeutung der Modelle und ihre Grenzen zu untersuchen. Dies sollte es dem Forschungsfeld ermöglichen, vom Bau von Systemen zu deren Nutzung überzugehen und auch neue Entdeckungen zu machen, anstatt bereits in vivo gemachte Beobachtungen wiederzuentdecken. Zusätzlich zur Bereitstellung von Organmodellen, die untersucht werden können, wird davon ausgegangen, dass Organoide auch von Nutzen sein werden, um die Mechanismen, die die Selbstorganisation von Organen ermöglichen, zu untersuchen. Selbstorganisierte Systeme in der biologischen und nicht-biologischen Welt können hierfür eine große Inspirationsquelle sein. 